\title{
Immunohistochemical Analysis of Hepatocellular and Adenocarcinoma in the Liver: MOC31 Compares Favorably with Other Putative Markers
}

Ana I. Porcell L., M.D., Barry R. De Young, M.D., Daniela M. Proca, M.D., Wendy L. Frankel, M.D. The Ohio State University Medical Center, Columbus, Ohio

Distinguishing hepatocellular carcinoma (HCC) from metastatic adenocarcinoma (MA) and cholangiocarcinoma (CC) can, at times, be difficult and sometimes requires immunohistochemical analysis. Recently, MOC31, an antibody directed against a cell surface glycoprotein, has been shown to be useful in separating HCC from both MA and CC; however, no study has compared MOC31 and other frequently used immunostains. We compare MOC31 with other commonly used immunostains for HCC, MA, and CC. Formalin-fixed, paraffin-embedded tissue sections from 57 previously characterized hepatic neoplasms (13 HCC, 14 CC, 3 combined HCCCC, and $27 \mathrm{MA}$ ) were immunostained with antibodies directed against MOC31, cytokeratin (CK) 7, CK20, $\alpha$-fetoprotein (AFP), polyclonal carcinoembryonic antigen, Ber-EP4, and Factor XIII-A. Two pathologists reviewed slides, and positivity was defined as more than $1 \%$ of cells staining with the appropriate pattern. Positive MOC31 immunostaining was seen in 0 of $13 \mathrm{HCC}, 13$ of $14 \mathrm{CC}, 3$ of 3 HCC-CC, and 27 of $27 \mathrm{MA}$; the staining was strong and diffuse. CK20 reactivity was observed in 0 of 13 HCC, 2 of 14 CC, 0 of 3 HCC-CC, and 12 of 27 MA; CK7 immunostained 4 of 13 HCC, 13 of 14 CC, 3 of 3 HCC-CC, and 15 of 27 MA; AFP was detected in 4 of 13 HCC and 2 of 3 HCC-CC, whereas all CC and $M A$ were negative; polyclonal carcinoembryonic antigen showed immunoreactivity in 12 of $13 \mathrm{HCC}$ and 3 of 3 HCC-CC in a canalicular pattern, whereas diffuse positivity was identified in 13 of $14 \mathrm{CC}$ and 26 of 27 MA; Ber-EP4 immunostained 1 of 13 HCC, 14 of 14 CC, 2 of 3 HCC-CC, and 26 of 27 MA; and Factor XIII-A was negative in all HCC, CC, and MA.

Copyright () 2000 by The United States and Canadian Academy of Pathology, Inc

VOL. 13, NO. 7, P. 773, 2000 Printed in the U.S.A.

Date of acceptance: January 7, 2000.

Presented in part at the American Society of Clinical Pathologists fall meeting, Washington, D.C., October 1998

Address reprint requests to: Wendy L. Frankel, M.D., The Ohio State University Medical Center, 401 East Doan Hall, 410 W 10th Avenue, Columbus, OH 43210; e-mail: frankel-1@medctr.osu.edu; fax: 614-2934715 .
MOC31 expression distinguished HCC from adenocarcinoma in 56 of 57 cases. AFP was specific for HCC but was not sensitive. CK7 and CK20 have limited utility in distinguishing HCC from CC or MA, and Factor XIII-A is not useful. Ber-EP4 staining was similar to MOC31, but one HCC did stain with Ber-EP4. Polyclonal CEA yields similar numerical results as MOC31, but the focal nature of the staining and occasional difficulty in evaluating the pattern can make interpretation problematic. We conclude that MOC31 should be a component of the immunohistochemical panel to distinguish HCC from CC and MA.

KEY WORDS: Cholangiocarcinoma, Cytokeratin profile, Hepatocellular carcinoma, Immunohistochemistry, Metastatic adenocarcinoma, MOC31.

Mod Pathol 2000;13(7):773-778

The histologic distinction between hepatocellular carcinoma (HCC) and adenocarcinoma (cholangiocarcinoma $[\mathrm{CC}]$ and metastatic adenocarcinoma [MA]) usually can be performed using routine hematoxylin and eosin-stained sections. In some instances, the differentiation can be difficult (Fig. 1) but is necessary when clinical and/or radiologic distinction cannot be made. Immunohistochemical panels have been used to separate these tumors with variable success. $\alpha$-Fetoprotein (AFP); polyclonal carcinoembryonic antigen (pCEA); and cytokeratins (CK) 7, 8, 18, 19, and 20 are among some of the more commonly used markers in these panels (1-8).

Recently, anti-MOC31, an antibody directed against a cell surface glycoprotein, was reported in our laboratory to distinguish between HCC and adenocarcinoma (9). MOC31 reacts with most benign and malignant epithelia and has been shown to be useful in panels to distinguish adenocarcinoma from mesothelioma (10-14). MOC31 has not been compared directly with other frequently used markers to distinguish between HCC and adenocarcinoma. In this study, we compare MOC31 with 


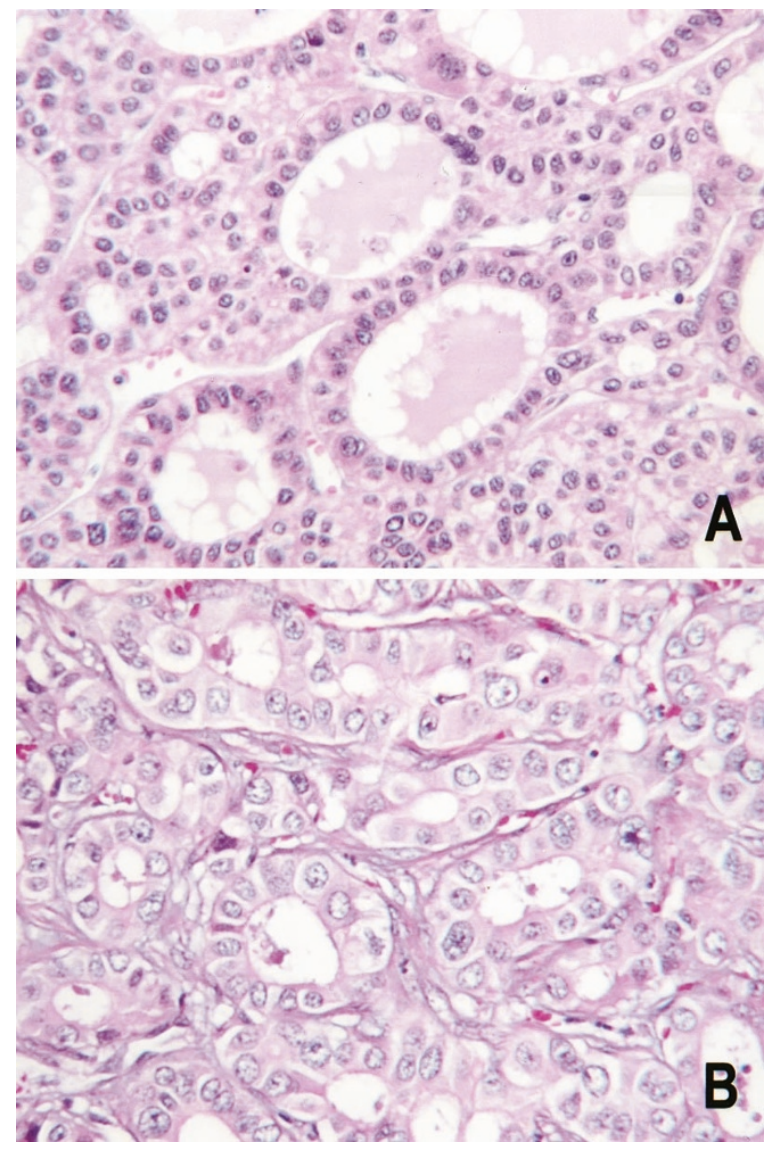

FIGURE 1. A, hepatocellular carcinoma, pseudoalveolar pattern (hematoxylin and eosin, $\times 320$ ). B, cholangiocarcinoma (hematoxylin and eosin, $\times 320$ ).

other immunohistochemical markers that are frequently used to distinguish between HCC and adenocarcinoma.

\section{MATERIALS AND METHODS}

Formalin-fixed, paraffin-embedded tissue blocks from 57 previously characterized hepatic neoplasms (13 HCC [including 5 pseudoalveolar], 14 CC, 3 combined HCC-CC, and 27 MA [10 colon, 6 breast, 6 pancreas, 3 gastrointestinal junction/gastric, 1 small intestine, and 1 ovary]) were retrieved from the archival files of the Ohio State University Medical Center, Columbus, Ohio.

Immunoperoxidase staining was performed on formalin-fixed, paraffin-embedded tissue sections cut at $3 \mu \mathrm{m}$ and dried for $1 \mathrm{~h}$ at $60^{\circ} \mathrm{C}$. The slides were deparaffinized in xylene and rehydrated through graded ethanol to water. Immunoperoxidase staining was performed on the Dako Autostainer (Carpinteria, CA). Antigen retrieval was performed with proteinase-K (S3020; DAKO) for 5 min at room temperature for MOC31 (antihuman epithelial-related antigen; DAKO, Carpinteria, CA; 1:40), CK7 (DAKO, 1:500), and Factor XIII-A (Dako, 1:400). Similar results can be obtained with heat-induced epitope retrieval for MOC31. Epitope enhancement using heatinduced epitope retrieval was used for CK20 (DAKO, 1:500), AFP (DAKO, 1:3000), pCEA (DAKO, 1:1250), and Ber-EP4 (MCF-7 cell line; Dako; 1:800). Slides were heated $\left(94\right.$ to $98^{\circ} \mathrm{C}$ ) in target retrieval solution (S1699; Dako) in a vegetable steamer for $30 \mathrm{~min}$ followed by a 5-min cool down period. Biotin blocking was performed with the DAKO biotin-blocking system. Sections were developed by using a modified avidin-biotin-peroxidase complex technique (Linked streptavidin-biotin + [MOC31, CK20, Ber-EP4, Factor XIII-A] and Linked streptavidin-biotin 2 [CK7, AFP, pCEA], K0675; Dako). After a brief rinse in tap water, the sections were counterstained with Harris hematoxylin and coverslipped using a synthetic mounting medium. Two pathologists reviewed slides, and positivity was defined as more than $1 \%$ of cells staining with the proper pattern of reactivity. Positive and negative controls stained appropriately.

\section{RESULTS}

The results of immunohistochemical stains are summarized in Table 1. MOC31 stained 0 of 13 HCC (Fig. 2A), 13 of 14 CC, 3 of 3 HCC-CC, and 27 of 27 $\mathrm{MA}$. The positive staining was strong and diffuse in a plasmalemmal pattern (Fig. 2B). In the HCC-CC cases, MOC31 decorated the glandular CC component but did not highlight the HCC areas.

TABLE 1. Immunohistochemical Staining in Hepatic Neoplasms

\begin{tabular}{|c|c|c|c|c|c|c|c|c|}
\hline Diagnosis & MOC31 & AFP & $\begin{array}{l}\text { pCEA } \\
\text { (cyto) }\end{array}$ & $\begin{array}{c}\text { pCEA } \\
\text { (canal) }\end{array}$ & CK7 & CK20 & Ber-EP4 & FXIII-A \\
\hline $\operatorname{HCC}(n=13)$ & $0 / 13$ & $4 / 13$ & $0 / 13$ & $12 / 13$ & $4 / 13$ & $0 / 13$ & $1 / 13$ & $0 / 13$ \\
\hline HCC-CC $(n=3)$ & $3 / 3$ & $2 / 3$ & $0 / 3$ & $3 / 3$ & $3 / 3$ & $0 / 3$ & $2 / 3$ & $0 / 3$ \\
\hline $\mathrm{CC}(n=14)$ & $13 / 14$ & $0 / 14$ & $13 / 14$ & $0 / 14$ & $13 / 14$ & $2 / 14$ & $14 / 14$ & $0 / 14$ \\
\hline Breast $(n=6)$ & $6 / 6$ & $0 / 6$ & $5 / 6$ & $0 / 6$ & $4 / 6$ & $0 / 6$ & $5 / 6$ & $0 / 6$ \\
\hline Pancreas $(n=6)$ & $6 / 6$ & $0 / 6$ & $6 / 6$ & $0 / 6$ & $6 / 6$ & $1 / 6$ & $6 / 6$ & $0 / 6$ \\
\hline $\operatorname{Sm~I~}(n=1)$ & $1 / 1$ & $0 / 1$ & $1 / 1$ & $0 / 1$ & $1 / 1$ & $0 / 1$ & $1 / 1$ & $0 / 1$ \\
\hline Ovary $(n=1)$ & $1 / 1$ & $0 / 1$ & $1 / 1$ & $0 / 1$ & $1 / 1$ & $0 / 1$ & $1 / 1$ & $0 / 1$ \\
\hline Colon $(n=10)$ & $10 / 10$ & $0 / 10$ & $10 / 10$ & $0 / 10$ & $0 / 10$ & $9 / 10$ & $10 / 10$ & $0 / 10$ \\
\hline GE-J $(n=3)$ & $3 / 3$ & $0 / 3$ & $3 / 3$ & $0 / 3$ & $3 / 3$ & $2 / 3$ & $3 / 3$ & $0 / 3$ \\
\hline
\end{tabular}

HCC, hepatocellular carcinoma; HCC-CC, combined hepatocellular carcinoma and cholangiocarcinoma; Sm I, small intestine; GE-J, gastroesophageal junction; CK, cytokeratin; pCEA, polyclonal carcinoembryonic antigen; AFP, $\alpha$-fetoprotein; cyto, cytoplasmic; canal, canalicular. 


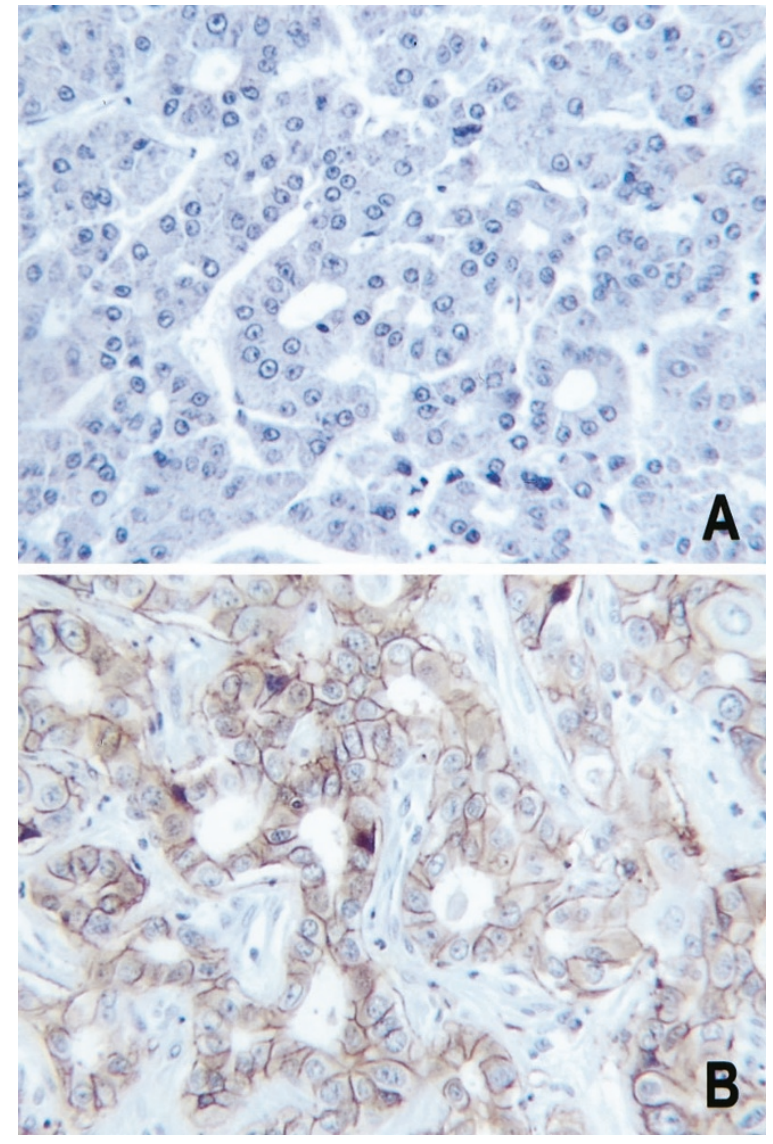

FIGURE 2. A, hepatocellular carcinoma, negative staining with MOC31 (anti-MOC31 with hematoxylin counterstain, $\times 320$ ). B, cholangiocarcinoma, positive staining with MOC31 (anti-MOC31 with hematoxylin counterstain, $\times 320$ ).

AFP-positive cells were identified in 4 of 13 HCC (Fig. 3) and 2 of 3 HCC-CC, whereas all of the CC and MA were uniformly negative. Immunostaining for AFP was focal in the positive cases. The predominant pattern of staining (canalicular or cytoplasmic) for pCEA is shown is Table 1. pCEA stained 12 of 13 HCC (Fig. 4A) and 3 of 3 HCC-CC in a canalicular pattern, and cytoplasmic positivity was iden-

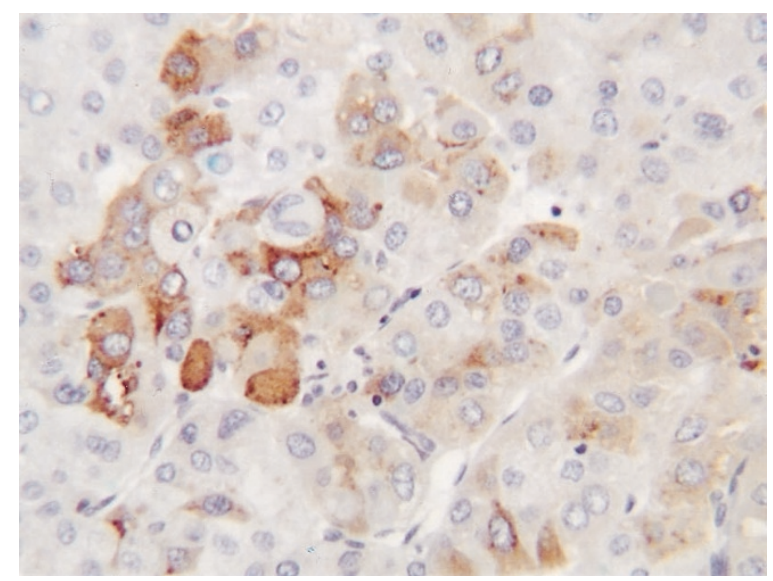

FIGURE 3. Hepatocellular carcinoma, focal positive staining with $\alpha$ fetoprotein (anti- $\alpha$-fetoprotein with hematoxylin counterstain, $\times 320$ ). tified in 13 of 14 CC and 26 of 27 MA (Fig. 4B). Interpretation of pCEA was difficult in some cases of adenocarcinomas because of a focal pattern of staining resembling canalicular staining (four cases; Fig. 4C). In addition, the pseudoalveolar HCC showed luminal staining in addition to a focal canalicular pattern (five cases; Fig. 4D). CK 7 stained 4 of 13 HCC, 13 of 14 CC, 3 of 3 HCC-CC, and 15 of 27 MA. CK 20 was observed in 0 of 13 HCC, 2 of 14 CC, 0 of 3 HCC-CC, and 12 of 27 MA. Ber-EP4 immunostained 1 of 13 HCC, 14 of 14 CC, 2 of 3 HCC-CC, and 26 of 27 MA. Factor XIII-A was uniformly negative in HCC, CC, and MA.

\section{DISCUSSION}

Adenocarcinomas from many sites commonly metastasize to the liver, and MA is the most common malignant tumor in the adult liver (15). As a result, consideration must be given to metastases in the differential diagnosis of a liver neoplasm. Usually, the distinction between HCC and either primary adenocarcinoma or MA can be made on the basis of clinical studies. In some instances, the pathologist is called on to differentiate histologically between these lesions. Routine hematoxylin and eosin sections and mucin stains may prove adequate in some cases. However, when limited material is available or when the tumor is poorly differentiated, various immunohistochemical panels have been used to aid in this distinction. The most commonly used markers include antibodies directed against AFP $(1,7)$, CEA $(1,2)$, and various cytokeratin subtypes $(3-6,8)$. Other markers that have been reported as useful include Factor XIII-A $(2,16)$, hepatitis B surface antigen (17), C-reactive protein (2), $\alpha$-1 antitrypsin (16), HepPar 1 (18), BerEP4 $(1,19)$, and molecular probes designed to identify albumin mRNA $(20,21)$.

MOC31 is a cell surface glycoprotein of unknown function, and the pattern of reaction is plasmalemmal. MOC31 is expressed on most normal and malignant epithelia. It has been reported to be a useful marker in the immunohistochemistry panel used to distinguish adenocarcinoma from malignant mesothelioma in multiple studies (10-14). One study reported strong expression of MOC31 in all 23 cases of adenocarcinoma evaluated. In contrast, only 1 of 23 mesothelioma cases was reported positive, albeit weakly (10). The efficacy of MOC31 in cytology specimens (cell-block) to differentiate adenocarcinoma from reactive mesothelial cells and mesothelioma has also been demonstrated (22). Our laboratory has previously reported the utility of MOC31 in distinguishing HCC from adenocarcinoma (9). In the present study, all adenocarcinomas stained diffusely and intensely with 

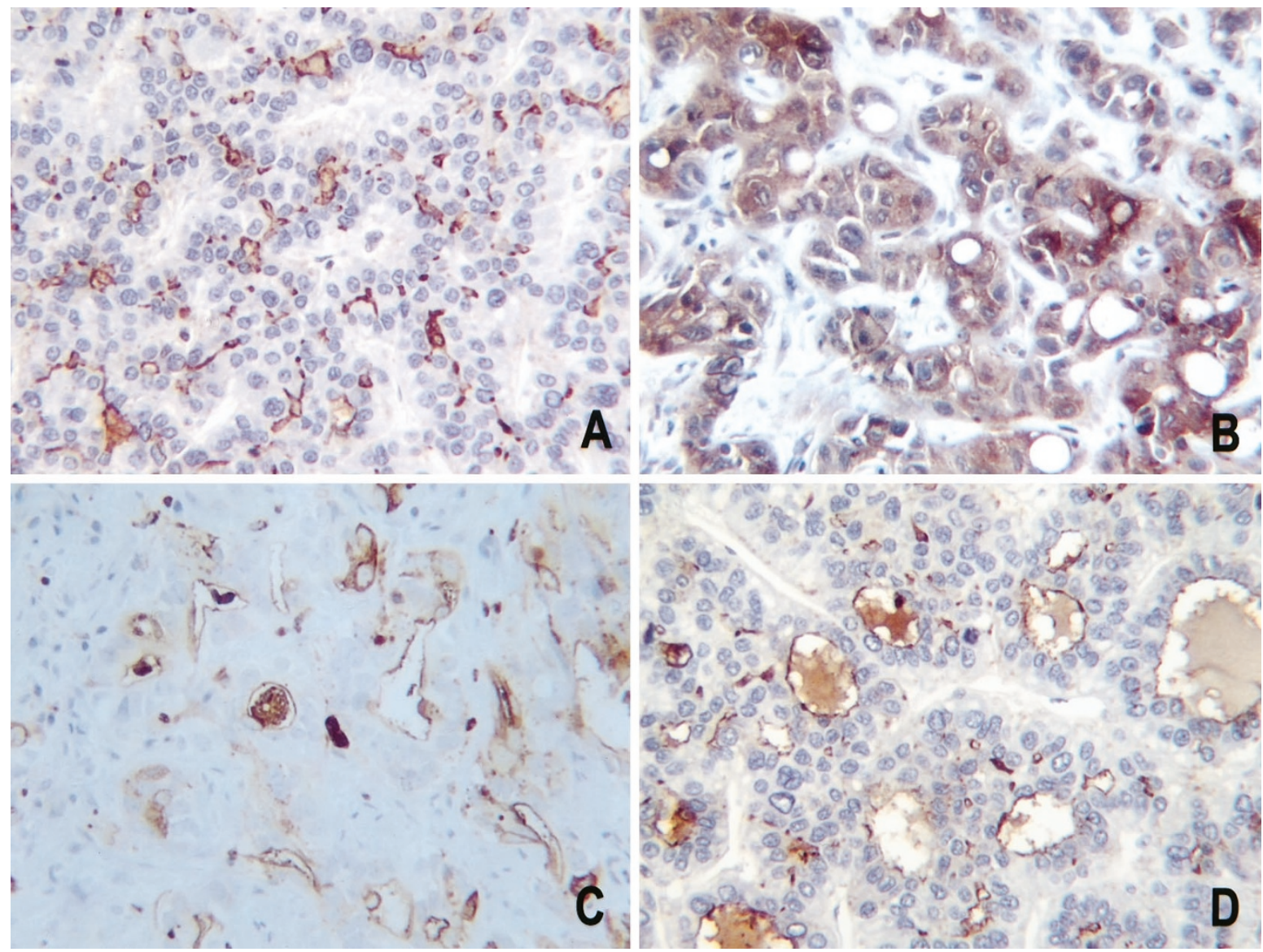

FIGURE 4. A, hepatocellular carcinoma, canalicular pattern of staining with polyclonal carcinoembryonic antigen (pCEA) (anti-pCEA with hematoxylin counterstain, $\times 320$ ). B, cholangiocarcinoma, positive cytoplasmic staining with pCEA (anti-pCEA with hematoxylin counterstain, $\times 320$ ). C, cholangiocarcinoma, difficult pattern of pCEA to interpret with focal pattern resembling canalicular staining (anti-pCEA with hematoxylin counterstain, $\times 320$ ). D, hepatocellular carcinoma, luminal pattern of staining with pCEA (anti-pCEA with hematoxylin counterstain, $\times 320$ ).

MOC31, yielding easy interpretation. In addition, 13 of 14 CC were decorated. All HCC, including the pseudoalveolar type, were uniformly negative. In the combined HCC-CC cases, the antibody highlighted the glandular component but did not decorate the HCC portion of the neoplasm.

In contrast to MOC31, there are some immunohistochemical stains that have been suggested as positive markers of HCC, such as HepPar 1, albumin mRNA, Factor XIII-A, and AFP. HepPar 1 is a monoclonal antibody that reacts against a hepatocyte-specific epitope, but the precise antigen is not known. Albumin mRNA is detected by in situ hybridization. These markers seem to be fairly specific for hepatocytes $(18,19)$, but HepPar 1 is not commercially available, and the detection of albumin mRNA requires in situ hybridization ability, which is expensive, time consuming, and not available in all laboratories. As a result, these markers were not evaluated in this study. Factor XIII is a protransglutaminase involved in hemostasis that is cleaved and leads to the formation of Factor XIII-A. Two studies have demonstrated that Factor XIII-A is useful for the identification of HCC $(2,16)$, whereas another investigation showed that HCC were uni- formly negative for this marker (23). In our study, all cases of HCC as well as adenocarcinoma were nonimmunoreactive with Factor XIII-A.

AFP is an oncofetal protein produced by hepatocytes. Positive immunostaining for AFP has been reported to be present in 2 to $61 \%$ of HCC (18). Expression of AFP can be focal and weak, leading to poor sensitivity, especially in small biopsies $(2,7)$. Expression of AFP may be related to tumor grade, with higher grade HCC expressing AFP more frequently than lower grade tumors. This tendency makes the marker useful, because high-grade HCC may lack a canalicular staining pattern with pCEA (1). AFP is not entirely specific for HCC as it can be expressed by benign liver lesions (alcoholic hepatitis, cirrhosis, and focal nodular hyperplasia) (17), as well as rare gastrointestinal tumors (24-26). In the present study, AFP lacked desired sensitivity; only 4 of 13 cases of HCC and 2 of 3 cases of HCC-CC stained for AFP. Because none of the adenocarcinomas was positive for AFP, it can be concluded that AFP, when positive, is a specific marker for HCC.

Expression of various cytokeratins has recently become a popular way to help distinguish primary 
sites of metastases because malignant cells usually maintain the cytokeratin profile of their "cells of origin." Adult hepatocytes express CK8 and 18, whereas bile duct epithelium expresses CK7, 8, 18, and 19 (4). Most HCC are negative for CK7 and 20 (8). CK20 is commonly expressed in gastrointestinal epithelia, urothelium, and Merkel cells (8). We found CK7 positivity in 4 of 13 cases of HCC, 3 of 3 HCC-CC, 13 of 14 CC, and 15 of 27 MA. These results agree with other studies that have demonstrated CK7 positivity in some HCC $(6,8)$. Although CK7 was more often identified in adenocarcinoma, positive staining is encountered in HCC. CK20 was negative in all cases of HCC and HCC-CC and positive in 2 of 14 CC. The lack of CK20 immunoreactivity in HCC concurs with the findings in other studies $(6,8)$. CK20 may help exclude HCC when it is positive, but negativity can be seen in HCC, CC, and MA.

CEA is an oncofetal protein that is widely used in surgical pathology. CEA is expressed in gastrointestinal, pancreatic, and biliary tract adenocarcinoma (27). A bile canalicular pattern of staining is considered typical and specific for liver, and this pattern results from cross-reactivity of pCEA with biliary glycoprotein 1 , normally present in bile canaliculi and bile ducts (1). The bile canalicular pattern of staining with pCEA in HCC has been reported in 60 to $90 \%$ of cases in the literature, and this pattern is seen more frequently in well- and moderately differentiated HCC than in poorly differentiated HCC (1). HCC, liver adenoma, focal nodular hyperplasia, and normal liver also exhibit a canalicular staining pattern with this antibody (17). The absence of bile canalicular staining does not entirely exclude HCC, as negative cases have been reported. Adenocarcinomas, in contrast to HCC, show a diffuse cytoplasmic pCEA staining pattern without canalicular accentuation (1).

In our study, pCEA stained 12 of 13 cases of HCC in a canalicular pattern and 39 of 41 cases of adenocarcinoma in a diffuse cytoplasmic pattern. However, difficulty was encountered, at times, in interpretation of the stain. Occasional cases of HCC that showed an overall bile canalicular pattern had foci of cytoplasmic or luminal (pseudoglandular areas) staining, and some cases of adenocarcinoma had areas that resembled a bile canalicular pattern. This was especially true in cases with strong staining. This variable staining pattern could lead to misinterpretation, especially when considering small specimens such as needle biopsies. This difficulty in interpretation of pCEA staining has been previously described. One study reported focal cytoplasmic pCEA in $41 \%$ of HCC and $71 \%$ of MA. A canalicular pattern was seen in $71 \%$ of HCC. Some HCC with an intense bile canalicular pattern also showed cytoplasmic staining, which was thought to represent a secondary phenomenon (1).

Ber-EP4 is an antibody directed against a cell surface glycoprotein present in non-neoplastic epithelial cells but is usually negative in hepatocytes, parietal cells, and the superficial layer of squamous epithelia. It has been used in panels to differentiate adenocarcinomas from mesotheliomas (19). In our study, all but one adenocarcinoma and all CC were immunoreactive with Ber-EP4. One of the 13 HCC was immunoreactive with Ber-EP4. Our results differ somewhat from a previous study that evaluated the utility of Ber-EP4 in distinguishing HCC from adenocarcinoma, which demonstrated anti-BerEP4 staining in $83 \%$ of MA and $36 \%$ of HCC (1). Additional investigation may help clarify the utility of Ber-EP4 in these cases.

MOC31 compared favorably with other routine markers used to differentiate HCC from adenocarcinoma. It is not useful for differentiating CC from MA or defining site of origin of metastases. It seems to possess a similar sensitivity and specificity as pCEA, but we believe that the staining is much easier to interpret. The staining was similar to that seen with Ber-EP4, but one HCC was immunoreactive with Ber-EP4. AFP suggests hepatocellular differentiation of tumors when positive but is negative in many cases of HCC. CK7 and Factor XIII-A were not helpful in distinguishing between HCC and adenocarcinomas, whereas CK20 was useful when positive. We confirm that CK7 and CK20 may have a role in suggesting the primary site of origin of metastatic tumors.

As with all neoplasms, immunohistochemistry is most useful when used in a panel. The results of this study suggest that the best panel to differentiate between HCC and adenocarcinoma, when necessary, should include MOC31. pCEA, AFP, and BerEP4 are also helpful. CK7 and CK20 may be useful when trying to determine primary site of a metastasis.

\section{REFERENCES}

1. Ma CK, Zarbo RJ, Frierson HF Jr, Lee MW. Comparative immunohistochemical study of primary and metastatic carcinomas of the liver. Am J Clin Pathol 1993;99:551-7.

2. Hurlimann J, Gardiol D. Immunohistochemistry in the differential diagnosis of liver carcinomas. Am J Surg Pathol 1991;15:280-8.

3. Johnson DE, Herndier BG, Medeiros LJ, Warnke RA, Rouse RV. The diagnostic utility of the keratin profiles of hepatocellular carcinoma and cholangiocarcinoma. Am J Surg Pathol 1988;12:187-97.

4. Van Eyken P, Sciot RAF, Paterson A, Callea F, Kew MC, Desmet VJ. Cytokeratin expression in hepatocellular carcinoma: an immunohistochemical study. Hum Pathol 1988; 19:562-8.

5. Maeda T, Adachi E, Kajiyama K, Sugimachi K, Tsuneyoshi M. Combined hepatocellular and cholangiocarcinoma: pro- 
posed criteria according to cytokeratin expression and analysis of clinicopathologic features. Hum Pathol 1995;26:95664 .

6. Maeda T, Kajiyama K, Adachi E, Takenaka K, Sugimachi K, Tsuneyoshi M. The expression of cytokeratins 7, 19, and 20 in primary and metastatic carcinomas of the liver. Mod Pathol 1996;9:901-9.

7. Brumm C, Schulze C, Charles K, Morohoshi T, Klöppel G. The significance of alpha-fetoprotein and other tumour markers in differential immunocytochemistry of primary liver tumours. Histopathology 1989;14:503-13.

8. Wang NP, Zee S, Zarbo RJ, Bacchi CE, Gown AM. Coordinate expression of cytokeratins 7 and 20 defines unique subsets of carcinomas. Appl Immunohistochem 1995;3:99-107.

9. Proca DM, Porcell AI, Niemann TH, De Young BR. MOC 31 immunoreactivity in primary and metastatic carcinoma of the liver. Appl Immunohistochem Mol Morphol 2000;5:120-5.

10. Sosolik RC, McGaughy VR, De Young BR. Anti-MOC31. A potential addition to the pulmonary adenocarcinoma versus mesothelioma immunohistochemistry panel. Mod Pathol 1997;10:716-9.

11. Ordonez NG. Value of the MOC-31 monoclonal antibody in differentiating epithelial pleural mesothelioma from lung adenocarcinoma. Hum Pathol 1998;29:166-9.

12. Edwards C, Oates J. OV632 and MOC-31 in the diagnosis of mesothelioma and adenocarcinoma: and assessment of their use in formalin-fixed and paraffin-wax embedded material. J Clin Pathol 1995;48:626-30.

13. Ryan PJ, Oates JL, Crocker J, Stableforth DE. Distinction between pleural mesothelioma and pulmonary adenocarcinoma using MOC31 in an asbestos sprayer. Respir Med 1997;91:57-60.

14. Ordonez NG. Role of immunohistochemistry in distinguishing epithelial peritoneal mesotheliomas from peritoneal and ovarian serous carcinomas. Am J Surg Pathol 1998;22:120314.

15. Pickren JW, Tsukada Y, Lane WW. Liver metastasis: analysis of autopsy data. In: Weiss L, Gilbert HA, editors. Liver metastasis. Boston: Hall Medical Publishers; 1982. p. 2-19.

16. Fucich LF, Cheles MD, Thung SN, Gerber MA, Marrogi AJ. Primary vs metastatic hepatic carcinoma. An immunohistochemical study of 34 cases. Arch Pathol Lab Med 1994;118: 927-30.
17. Koelma IA, Nap M, Huitema S, Krom RAF, Houthoff HJ. Hepatocellular carcinoma, adenoma, and focal nodular hyperplasia. Comparative histopathologic study with immunohistochemical parameters. Arch Pathol Lab Med 1986;110:1035-40.

18. Minervini MI, Demetris AJ, Lee RG, Carr BI, Marariaga J, Nalesnik MA. Utilization of hepatocyte-specific antibody in the immunocytochemical evaluation of liver tumors. Mod Pathol 1997;10:686-92.

19. Latza U, Niedobitek G, Schwarting R, Nekarda H, Stein H. Ber-EP4: new monoclonal antibody which distinguishes epithelia from mesothelia. J Clin Pathol 1990;43:213-9.

20. D'Errico A, Baccarini P, Fiorentino M, Ceccarelli C, Bonazzi C, Ponzetto A, et al. Histogenesis of primary liver carcinomas: strengths and weaknesses of cytokeratin profile and albumin mRNA detection. Hum Pathol 1996;27:599-604.

21. Krishna M, Lloyd RV, Batts KP. Detection of albumin messenger RNA in hepatic and extrahepatic neoplasms. A marker of hepatocellular differentiation. Am J Surg Pathol 1997;21:147-52.

22. Morgan RL, McGaughy VR, De Young BR, Niemann TH. Anti MOC 31 evaluation of cytologic fluids: adenocarcinoma versus reactive mesothelial cells. Cancer Cytopathol 1999;87: $390-4$.

23. Leong AS, Somunen RT, Tsui WM, Liew CT. Hep Par I and selected antibodies in the immunohistological distinction of hepatocellular carcinoma from cholangiocarcinoma, combined tumours and metastatic carcinoma. Histopathology 1998;33:318-24.

24. Kodama T, Kameya T, Hirota T, Shimosato Y, Ohkura H, Mukojima T, et al. Production of alpha-fetoprotein, normal serum proteins, and human chorionic gonadotropin in stomach cancer: histologic and immunohistochemical analyses of 35 cases. Cancer 1981;48:1647-55.

25. Yoshino I, Hayashi I, Yano T, Takai E, Mizutani K, Ichinose Y. Alpha-fetoprotein-producing adenocarcinoma of the lung. Lung Cancer 1996;15:125-30.

26. Kawamoto S, Hiraoka T, Kanemitsu K, Kimura M, Miyauchi Y, Takeya M. Alpha-fetoprotein-producing pancreatic cancer-a case report and review of 28 cases. Hepatogastroenterology 1992;39:282-6.

27. Carroza MJ, Calafata SA, Edmonds PR. Immunocytochemical localization of polyclonal carcinoembryonic antigen in hepatocellular carcinomas. Acta Cytologica 1991;35:221-4. 